\title{
Cluster-state preparation and multipartite entanglement analyzer with fermions
}

\author{
X. L. Zhang ${ }^{1,2}$ \& M. Feng ${ }^{1}$, and K. L. Gao ${ }^{1}$ \\ ${ }^{1}$ State Key Laboratory of Magnetic Resonance and Atomic and Molecular Physics, \\ Wuhan Institute of Physics and Mathematics, Chinese Academy of Sciences, \\ Wuhan 430071, People's Republic of China and \\ ${ }^{2}$ Graduate School of the Chinese Academy of Science, Bejing 100049, People's Republic of China
}

Abstract

\begin{abstract}
Quantum cluster states and entangled state analyzers are essential to measurement-based quantum computing. We propose to generate a quantum cluster-state and to make multipartite entanglement analyzer by using noninteracting free electrons or conduction electrons in quantum dots, based on polarizing beam splitters, charge detectors and single-spin rotations. Our schemes are deterministic without the need of qubit-qubit interaction.
\end{abstract}

PACS numbers: 03.67.Lx, 03.67.Mn, 03.67.Pp

Quantum computers own great advantages over existing computers to solve classically intractable problems and to speed up some tractable solutions [1, 2, 3]. Most current researches are exploring quantum computing based on externally controlled qubit-qubit interaction. While there are a kind of alternative quantum computing proposals based on qubit measurements.

The quantum cluster state [4], a special multipartite entangled state, is the key ingredient in a measurementbased quantum computing, i.e., one-way quantum computing [5]. With a cluster state of an array of qubits, we can carry out expected quantum gates by some singlequbit operations and detections. In a lattice structure, for any site $a$ of the lattice, the cluster state $\left|\phi_{\{k\}}\right\rangle_{C}$ obeys the set of eigenvalue equations $K^{(a)}\left|\phi_{\{k\}}\right\rangle_{C}=$ $(-1)^{\kappa_{a}}\left|\phi_{\{k\}}\right\rangle_{C}$, with the corresponding operator $K^{(a)}=$ $\sigma_{x}^{(a)} \underset{b \in n g h b(a)}{\otimes} \sigma_{z}^{(b)}$, where $\operatorname{nghb}(\mathrm{a})$ specifies the sites of all the neighbors of the site $a$ and $\kappa_{a} \in\{0,1\}$. The operators $K^{(a)}$ with $\{a \in$ lattice $\}$ form a complete family of commuting operators on the lattice. It has been shown that some physical systems such as quantum dots, optical photons and cavity quantum electrodynamics (QED) are suitable for preparation of cluster states [6, 7, 8, 9].

Alternatively, if we have Bell - state measurements (or say, analyzers) and some initial sources of entanglements, quantum gates necessary for universal quantum computing are also available. A typical example for this quantum computing is to use linear optical elements, e.g., polarizing beam splitters, as proposed in [10, 11]. It is generally believed that for measurement-based quantum computing, either full Bell-state measurements in combination with some initial source of entanglement or partial Bellstate measurements are sufficient for a universal quantum computing [12, 13].

In this work, we report how to generate cluster states and to make analyzers for multipartite entangled states with noninteracting free electrons or conduction electrons

*Electronic address: xili-zhang@hotmail.com in quantum dots, by using beam splitters and single-spin rotations. Our work is inspired by recent work 12, 14], which show that quantum information processing with such electrons could be simpler but more powerful than by using photons. However, a previous no-go theorem [15, 16] declared that the operators with beam splitters and single-spin rotations, can only achieve universal quantum computing in noninteracting boson systems instead of in fermions'. Nevertheless, Ref. [17] shows that entangled states can be created probabilistically using beam splitters and position detections for bosons as well as for fermions. Recently, more specific schemes 12, 14] were proposed by using noninteracting free electrons or conduction electrons in quantum dots to carry out universal quantum computing with beam splitters and single-spin rotations. The present paper consists of two parts. In the first part, we first describe how to construct the cluster states with noninteracting electrons using polarizing beam splitters and single-spin rotations, assisted by parity check on the qubits. In contrast to the design in [18] with success probability of 0.25 by using polarized photons, our scheme is deterministic without using any prior entanglement necessary in former parametric-down-conversion-based protocols. In the second part of the paper, we describe entanglement analyzers for multipartite states which would be useful for quantum communication with multipartite states and for judging multipartite entanglement [19, 20].

Like in $[12,14]$, we encode the qubits into the spin degrees of freedom of the noninteracting electrons. We first show in Fig. 1 how to realize a controlled phase (Cphase) gate using the encoder designed in [12], which is modified from the Fig. 3 of [12]. Based on this design, we could realize a cluster state in the following. We consider $\mathrm{N}$ noninteracting electrons in Fig. 2 prepared initially in a product state $\left|\phi_{0}\right\rangle=\otimes_{j}|+\rangle_{j}$, where indexes $j=1,2,3, \cdots$ refer to the sites of the atoms and $|+\rangle_{j}$ is the eigenstate of $\sigma_{x}^{(j)}$ with the eigenvalue 1. A straightforward deduction yields that, when $P_{j}=1$, the pair 
states of 1-2, 3-4, and so on, will collapse to

$$
\frac{1}{\sqrt{2}}(|00\rangle+|11\rangle) \text {. }
$$

After Hadamard transformations are performed on the second qubits of each pairs, Eq. (1) becomes

$$
\frac{1}{2}(|00\rangle+|01\rangle+|10\rangle-|11\rangle)
$$

This is a typical quantum phase gate for each electron pairs. For example, when the charge detectors $\mathrm{P}_{1}$ and $\mathrm{P}_{2}$ are both equal to 1 , the output qubits $1,2,3$ and $4^{\prime}$ fall to

$$
\begin{aligned}
|\Psi\rangle_{1234^{\prime}} & =1 / 4(|0000\rangle+|0001\rangle+|0010\rangle-|0011\rangle \\
& +|0100\rangle+|0101\rangle-|0110\rangle+|0111\rangle \\
& +|1000\rangle+|1001\rangle+|1010\rangle-|1011\rangle \\
& -|1100\rangle-|1101\rangle+|1110\rangle-|1111\rangle),
\end{aligned}
$$

which can also be rewritten to be a standard form of the cluster state,

$$
\begin{aligned}
|\Psi\rangle_{1234^{\prime}} & =1 / 4\left(|0\rangle_{1}+|1\rangle_{1} \sigma_{z}^{2}\right) \otimes\left(|0\rangle_{2}+|1\rangle_{2} \sigma_{z}^{3}\right) \\
& \otimes\left(|0\rangle_{3}+|1\rangle_{3} \sigma_{z}^{4}\right) \otimes\left(|0\rangle_{4}+|1\rangle_{4}\right) .
\end{aligned}
$$

If $\mathrm{P}_{1}$ and $\mathrm{P}_{2}$ are not both equal to 1 , a single-qubit operation is needed after above operations to realize the required state in Eq. (4). For example, in Table 1, where the first row means the case when $P_{1}$ gets the value 0 or 1 and the first column means the case of $P_{2}=0$ or $1 ; \sigma_{x 2^{\prime}}$ and $\sigma_{x 3^{\prime}}$ are spin-flip operations on the qubits output from the ports Nos. $2^{\prime}$ and $3^{\prime}$, respectively, and $I$ is the identity operator. It is easy to check that above idea can be extended to generation of N-electron cluster state by the design shown in Fig. 2. For any $\mathrm{P}_{j}=0$ we just need a spin-flip $\left(\sigma_{x}\right)$ operation on one of the electron spins in the control-out arm of the corresponding encoders.

Now we start to construct analyzers for multipartite entangled states. In Fig. 3 we show how multipartite entangled states can be distinguished using blocks of the encoder. In the full Bell-state analyzer shown in Fig. 3 (a), the different outputs of $P_{j}(\mathrm{j}=1,2)$ correspond to different Bell-states, where $P_{1}=1 \rightarrow\left|\Phi^{+}\right\rangle$or $\left|\Phi^{-}\right\rangle$; $P_{1}=0 \rightarrow\left|\Psi^{+}\right\rangle$or $\left|\Psi^{-}\right\rangle, P_{2}=1 \rightarrow\left|\Phi^{+}\right\rangle$or $\left|\Psi^{+}\right\rangle$; $P_{2}=0 \rightarrow\left|\Phi^{-}\right\rangle$or $\left|\Psi^{-}\right\rangle$, with $\left|\Phi^{ \pm}\right\rangle$and $\left|\Psi^{ \pm}\right\rangle$being, respectively, the Bell-states $\left|\Phi^{ \pm}\right\rangle=\frac{1}{\sqrt{2}}(|00\rangle \pm|11\rangle)$ and $\left|\Psi^{ \pm}\right\rangle=\frac{1}{\sqrt{2}}(|01\rangle \pm|10\rangle)$. For example, if we have $P_{1}=1$ and $P_{2}=1$, we know the electrons passing our analyzer to be in $\left|\Phi^{+}\right\rangle$.

In the case of a three-electron entangled state shown in Fig. 3 (b), after qubits 1 and 2 go through the encoder $P_{1}$, we can distinguish $\left\{g_{1}, g_{2}\right\}$ from $\left\{g_{3}, g_{4}\right\}$ when $P_{1}=$ 1 by a parity check on the detected pairwise 1-2, where $g_{1}=|\psi\rangle_{i, i i}, g_{2}=|\psi\rangle_{i i i, i v}, g_{3}=|\psi\rangle_{v, v i}, g_{4}=|\psi\rangle_{v i i, v i i i}$ by using following equations,

$$
\begin{aligned}
|\psi\rangle_{i, i i} & =\frac{1}{\sqrt{2}}\left(|000\rangle_{123} \pm|111\rangle_{123}\right) \\
& =\left|\Phi^{+}\right\rangle_{12}| \pm\rangle_{3}+\left|\Phi^{-}\right\rangle_{12}|\mp\rangle_{3}, \\
|\psi\rangle_{i i i, i v} & =\frac{1}{\sqrt{2}}\left(|110\rangle_{123} \pm|001\rangle_{123}\right) \\
& =\left|\Phi^{+}\right\rangle_{12}| \pm\rangle_{3}-\left|\Phi^{-}\right\rangle_{12}|\mp\rangle_{3}, \\
|\psi\rangle_{v, v i} & =\frac{1}{\sqrt{2}}\left(|010\rangle_{123} \pm|101\rangle_{123}\right) \\
& =\left|\Psi^{+}\right\rangle_{12}| \pm\rangle_{3}+\left|\Psi^{-}\right\rangle_{12}|\mp\rangle_{3}, \\
|\psi\rangle_{v i i, v i i i} & =\frac{1}{\sqrt{2}}\left(|100\rangle_{123} \pm|011\rangle_{123}\right) \\
& =\left|\Psi^{+}\right\rangle_{12}| \pm\rangle_{3}-\left|\Psi^{-}\right\rangle_{12}|\mp\rangle_{3} .
\end{aligned}
$$

If $\mathrm{P}_{1}=0$, with a $\sigma_{x}$ operation on one of the output electrons from the encoder $\mathrm{P}_{1}$, we can do the same job as in the case of $\mathrm{P}_{1}=1$. Similarly, after electrons go through the encoder $\mathrm{P}_{2}$, we can distinguish $\left\{g_{1}, g_{4}\right\}$ from $\left\{g_{2}, g_{3}\right\}$. So up to now we have separated the eight three-qubit entangled states into four groups, i.e., $g_{1}, g_{2}, g_{3}, g_{4}$. In order to distinguish the two states (i.e., the + state from state) in each groups, we have to perform measurements on each group. For example, to distinguish the state $\frac{1}{\sqrt{2}}(|000\rangle+|111\rangle)$ from $\frac{1}{\sqrt{2}}(|000\rangle-|111\rangle)$, if we get a click in the basis $|+\rangle$ and $\mathrm{P}_{3}=1$, or get a click in $|-\rangle$ and $\mathrm{P}_{3}=0$, we have the state $\frac{1}{\sqrt{2}}(|000\rangle+|111\rangle)$. Otherwise we have $\frac{1}{\sqrt{2}}(|000\rangle-|111\rangle)$. Although each measurement would destroy a qubit, as the eight entangled states can be completely distinguished, the device in Fig. 3 (b) would be useful for teleportation and superdense coding with tripartite states [19, 20].

The idea can be generalized to many-electron cases, which would be more complicated but still deterministic. We show in Fig. 3 (c) an example for a four-electron entanglement analyzer. By checking the parity of the two pairs 1-2 and 3-4, along with the readouts of $\mathrm{P}_{1}$ and $\mathrm{P}_{2}$, we can separate the four-qubit entangled states into four sets of groups, as shown in Table 2, by using following 
Eq. (6),

$$
\begin{aligned}
& |\psi\rangle_{i}=\frac{1}{\sqrt{2}}\left(|0000\rangle_{1234} \pm|1111\rangle_{1234}\right) \\
& =\left\{\begin{array}{l}
\frac{1}{\sqrt{2}}\left(\left|\Phi^{+}\right\rangle_{12}\left|\Phi^{+}\right\rangle_{34}+\left|\Phi^{-}\right\rangle_{12}\left|\Phi^{-}\right\rangle_{34}\right) \text { for }+ \text { state } \\
\frac{1}{\sqrt{2}}\left(\left|\Phi^{+}\right\rangle_{12}\left|\Phi^{-}\right\rangle_{34}+\left|\Phi^{-}\right\rangle_{12}\left|\Phi^{+}\right\rangle_{34}\right) \text { for - state }
\end{array}\right. \\
& |\psi\rangle_{i i}=\frac{1}{\sqrt{2}}(|0001\rangle \pm|1110\rangle) \\
& =\left\{\begin{array}{l}
\frac{1}{\sqrt{2}}\left(\left|\Phi^{+}\right\rangle_{12}\left|\Psi^{+}\right\rangle_{34}+\left|\Phi^{-}\right\rangle_{12}\left|\Psi^{-}\right\rangle_{34}\right) \text { for }+ \text { state } \\
\frac{1}{\sqrt{2}}\left(\left|\Phi^{+}\right\rangle_{12}\left|\Psi^{-}\right\rangle_{34}+\left|\Phi^{-}\right\rangle_{12}\left|\Psi^{+}\right\rangle_{34}\right) \text { for - state }
\end{array}\right. \\
& |\psi\rangle_{i i i}=\frac{1}{\sqrt{2}}(|0010\rangle \pm|1101\rangle) \\
& =\left\{\begin{array}{l}
\frac{1}{\sqrt{2}}\left(\left|\Phi^{+}\right\rangle_{12}\left|\Psi^{+}\right\rangle_{34}-\left|\Phi^{-}\right\rangle_{12}\left|\Psi^{-}\right\rangle_{34}\right) \text { for }+ \text { state } \\
\frac{1}{\sqrt{2}}\left(\left|\Phi^{+}\right\rangle_{12}\left|\Psi^{-}\right\rangle_{34}-\left|\Phi^{-}\right\rangle_{12}\left|\Psi^{+}\right\rangle_{34}\right) \text { for - state }
\end{array}\right. \\
& |\psi\rangle_{i v}=\frac{1}{\sqrt{2}}(|0100\rangle \pm|1011\rangle) \\
& =\left\{\begin{array}{l}
\frac{1}{\sqrt{2}}\left(\left|\Psi^{+}\right\rangle_{12}\left|\Phi^{+}\right\rangle_{34}+\left|\Psi^{-}\right\rangle_{12}\left|\Phi^{-}\right\rangle_{34}\right) \text { for }+ \text { state } \\
\frac{1}{\sqrt{2}}\left(\left|\Psi^{+}\right\rangle_{12}\left|\Phi^{-}\right\rangle_{34}+\left|\Psi^{-}\right\rangle_{12}\left|\Phi^{+}\right\rangle_{34}\right) \text { for - state }
\end{array}\right.
\end{aligned}
$$

$$
\begin{aligned}
& |\psi\rangle_{v}=\frac{1}{\sqrt{2}}(|1000\rangle \pm|0111\rangle) \\
& =\left\{\begin{array}{l}
\frac{1}{\sqrt{2}}\left(\left|\Psi^{+}\right\rangle_{12}\left|\Phi^{+}\right\rangle_{34}-\left|\Psi^{-}\right\rangle_{12}\left|\Phi^{-}\right\rangle_{34}\right) \text { for }+ \text { state } \\
\frac{1}{\sqrt{2}}\left(\left|\Psi^{+}\right\rangle_{12}\left|\Phi^{-}\right\rangle_{34}-\left|\Psi^{-}\right\rangle_{12}\left|\Phi^{+}\right\rangle_{34}\right) \text { for - state }
\end{array}\right. \\
& |\psi\rangle_{v i}=\frac{1}{\sqrt{2}}(|0011\rangle \pm|1100\rangle) \\
& =\left\{\begin{array}{l}
\frac{1}{\sqrt{2}}\left(\left|\Phi^{+}\right\rangle_{12}\left|\Phi^{+}\right\rangle_{34}-\left|\Phi^{-}\right\rangle_{12}\left|\Phi^{-}\right\rangle_{34}\right) \text { for }+ \text { state } \\
\frac{1}{\sqrt{2}}\left(\left|\Phi^{+}\right\rangle_{12}\left|\Phi^{-}\right\rangle_{34}-\left|\Phi^{-}\right\rangle_{12}\left|\Phi^{+}\right\rangle_{34}\right) \text { for - state }
\end{array}\right. \\
& |\psi\rangle_{v i i}=\frac{1}{\sqrt{2}}(|0101\rangle \pm|1010\rangle) \\
& =\left\{\begin{array}{l}
\frac{1}{\sqrt{2}}\left(\left|\Psi^{+}\right\rangle_{12}\left|\Psi^{+}\right\rangle_{34}+\left|\Psi^{-}\right\rangle_{12}\left|\Psi^{-}\right\rangle_{34}\right) \text { for }+ \text { state } \\
\frac{1}{\sqrt{2}}\left(\left|\Psi^{+}\right\rangle_{12}\left|\Psi^{-}\right\rangle_{34}+\left|\Psi^{-}\right\rangle_{12}\left|\Psi^{+}\right\rangle_{34}\right) \text { for - state }
\end{array}\right. \\
& |\psi\rangle_{v i i i}=\frac{1}{\sqrt{2}}(|1001\rangle \pm|0110\rangle) \\
& =\left\{\begin{array}{l}
\frac{1}{\sqrt{2}}\left(\left|\Psi^{+}\right\rangle_{12}\left|\Psi^{+}\right\rangle_{34}-\left|\Psi^{-}\right\rangle_{12}\left|\Psi^{-}\right\rangle_{34}\right) \text { for }+ \text { state } \\
\frac{1}{\sqrt{2}}\left(\left|\Psi^{+}\right\rangle_{12}\left|\Psi^{-}\right\rangle_{34}-\left|\Psi^{-}\right\rangle_{12}\left|\Psi^{+}\right\rangle_{34}\right) \text { for - state }
\end{array}\right.
\end{aligned}
$$

where \pm state means $(|\cdots\rangle \pm|\cdots\rangle) / \sqrt{2}$, respectively. Another encoder $\mathrm{P}_{3}$ would further divide the four sets of groups into eight groups, i.e., $|\psi\rangle_{j}(\mathrm{j}=\mathrm{i}$, ii $\cdots$ viii). In order to completely distinguish the sixteen four-qubit entangled states, we have to make two measurements, as shown in Fig. 3 (c).

To achieve our proposal experimentally, we may encode the qubits into the spin degrees of freedom of, for example, the conduction electrons in a quantum dot system. A recent scheme 14] for such conduction electrons is proposed to convert spin parity into charge information by resonant tunneling between two dots when the spins are antiparallel. Moreover, both the beam splitters and the charge detectors required in our scheme have been experimentally achieved 21, 22, 23, 24] by means of the point contacts in two-dimensional electron gas. Since it only makes a parity check, the charge detector in our case can be realized by using the point contact made of a quantum dot with a resonant conductance. According to whether it is resonant or off-resonant, the detector can distinguish occupation number one from occupation number 0 or two. However, the great experimental challenge for charge detector is the time resolved detection required for the ballistic electrons, which at present is longer than our requirement by several orders [24]. We expect that this requirement could be met in the near future with the advance of the techniques in this respect.

In conclusion, we have presented schemes for fermions to deterministically generate cluster states and to deterministically distinguish multipartite entanglement based on polarizing beam splitters and single-spin rotations in combination with the charge detectors. The generation of the cluster states would be useful for one-way quantum computing with fermions, and the construction of analyzers for multipartite entangled state provides a potential way for fermionic quantum information processing with multipartite entangled states.

This work is supported in part by National Natural Science Foundation of China under Grant Nos. 10474118 and 10274093 , and partly by the National Fundamental Research Program of China under Grant Nos. 2001CB309309 and 2005CB724502.
[1] D. Deutsch and R. Jozsa, Proc. R. Soc. London, Ser. A 439, 553 (1992).

[2] A. Ekert and R. Jozsa, Rev. Mod. Phys. 68, 733 (1996).

[3] L.K. Grover, Phys. Rev. Lett. 79, 325 (1997); 79, 4709 (1997); 804329 (1998).

[4] H. J. Briegel and R. Raussendorf, Phys. Rev. Lett. 86, 910 (2001).

[5] R. Raussendorf and H. J. Briegel, Phys. Rev. Lett. 86, 5188 (2001).

[6] M. Borhani and D. Loss, Phys. Rev. A 71, 034308 (2005).
[7] P. Walther et al, Nature (London) 434, 169 (2005).

[8] D.E. Browne and T. Rudolph, Phys. Rev. Lett. 95, 010501 (2005).

[9] X.B. Zou and W. Mathis, Phys. Rev. A 72, 013809 (2005).

[10] E. Knill, R. Laflamme, and G.J. Milburn, Nature 409, 46 (2001).

[11] M.A. Nielsen and I.L. Chuang, Quantum Computation and Quantum Information (Cambridge University Press, Cambridge, England, 2000). 
[12] C.W.J. Beenakker, D.P. DiVincenzo, C. Emary, and M. Kindermann, Phys. Rev. Lett. 93, 020501 (2004).

[13] D. Gottesman and I.L. Chuang, Nature 402, 390 (1999).

[14] H.A. Engel and D. Loss, Science 309, 586 (2005).

[15] B.M. Terhal and D.P. DiVincenzo, Phys. Rev. A 65, 032325 (2002).

[16] E. Knill, quant-ph/0108033

[17] S. Bose and D. Home, Phys. Rev. Lett. 88, 050401 (2002).

[18] X.B. Zou and W. Mathis, Phys. Rev. A 71, 032308 (2005).

[19] V.N. Gorbachev, A.I. Trubilko, J. Exp. Theor.Phys. 91, 894 (2000); Zh. Eksp. Teor. Fiz. 91, 1036 (2000).

[20] H.J. Lee, D. Ahn, and S.W. Hwang, Phys. Rev. A 66, 024304 (2002).

[21] M. Henny et al, Science 284, 296 (1999).

[22] W.D. Oliver et al, Science 284, 299 (1999).
[23] E. Buks et al, Nature (London) 391, 871 (1998).

[24] J.M. Elzerman et al, Appl. Phys. Lett. 84, 4617 (2004).

Table. 1 The corresponding single-qubit operation for different values of $P_{1}$ and $P_{2}$ in preparation of a cluster state.

\begin{tabular}{|l|l|l|}
\hline$P_{2} \backslash P_{1}$ & 0 & 1 \\
\hline 0 & $\sigma_{x 2^{\prime}} \otimes \sigma_{x 3^{\prime}}$ & $I \otimes \sigma_{x 3^{\prime}}$ \\
\hline 1 & $\sigma_{x 2^{\prime}} \otimes I$ & $I \otimes I$ \\
\hline
\end{tabular}

Table. 2 Parity check for four-qubit entanglement analyzer.

\begin{tabular}{|l|l|l|}
\hline$P_{2} \backslash P_{1}$ & 0 & 1 \\
\hline 0 & $|\psi\rangle_{v i i},|\psi\rangle_{v i i i}$ & $|\psi\rangle_{i i},|\psi\rangle_{i i i}$ \\
\hline 1 & $|\psi\rangle_{i v},|\psi\rangle_{v}$ & $|\psi\rangle_{i},|\psi\rangle_{v i}$ \\
\hline
\end{tabular}

